\title{
Anti-Inflamm-Aging Effects of Long-Term Caloric Restriction via Overexpression of SIGIRR to Inhibit NF-kB Signaling Pathway
}

\author{
Xiao-meng Xu Yi-Chun Ning Wen-juan Wang Jie-qiong Liu Xue-yuan Bai \\ Xue-feng Sun Guang-yan Cai Xiang-mei Chen
}

Department of Nephrology, Chinese PLA General Hospital, Chinese PLA Institute of Nephrology, State Key Laboratory of Kidney Diseases, National Clinical Research Center for Kidney Diseases, Beijing, China

\section{Key Words}

Caloric restriction $\bullet$ Inflammation $•$ Aging $•$ Kidney $\bullet$ SIGIRR $\bullet N F-\kappa B$

\begin{abstract}
Background: Chronic inflammation is thought to be a determinant of the aging rate and longevity. Caloric restriction (CR) attenuates age-related increases in the systemic levels of several pro-inflammatory mediators, but the anti-inflammatory mechanisms of $C R$ in the aging process remain unclear. Methods: Fisher 344 rats in a CR group were fed an amount of food corresponding to $60 \%$ of that fed to an ad libitum-fed (AL) group for 8 months. Biochemical analyses and renal pathological grading were used to analyze physiological status. Important signaling molecules in the Toll-like receptor/nuclear factor kappa-light-chainenhancer of activated $B$ cells (TLR/NF-KB) pathway were also analyzed by western blotting, immunofluorescence and immunohistochemistry. Results: 1) Compared with AL feeding, CR decreased aging-mediated increases in both biochemical marker levels and renal pathological grading. 2) Single immunoglobulin IL-1 (IL-1)-related receptor (SIGIRR) expression decreased with increasing age, but CR led to overexpression. 3) The expression of TLR4 was significantly higher in the CR group than in the AL group. 4) SIGIRR overexpression decreased the expression of the adaptor molecules myeloid differentiation factor 88 (MyD88), IL-1 receptor-associated kinase 4 (IRAK4) and tumor necrosis factor receptor-associated factor 6 (TRAF6). 5) The levels of the inflammatory markers phospho-IKB $\alpha$ and phospho-NF-KB p65 decreased in the CR group. Conclusions: The inflammatory response might be alleviated by SIGIRR via blockade of the TLR4/NF-KB signaling pathway. Therefore, CR can decrease inflammation via SIGIRR overexpression, and SIGIRR might be a new target to delay aging.
\end{abstract}

Dr. Guang-yan Cai,

KARGER 125
Department of Nephrology, Chinese PLA General Hospital, Chinese PLA Institute of Nephrology, State Key Laboratory of Kidney Diseases, National Clinical Research Center for Kidney Diseases, 28, Fuxing Road, HaiDian District, Beijing 100853 (China) Tel. +86-10-66935462; Fax +86-10-6813 0297; E-Mail caiguangyan@sina.com 


\section{Cellular Physiology Cell Physiol Biochem 2015;37:1257-1270 \begin{tabular}{l|l} 
and Biochemistry & $\begin{array}{l}\text { DOI: 10.1159/000430248 } \\
\text { Published online: October 05, } 2015\end{array}$ \\
\hline
\end{tabular} \\ Xu et al.: Anti-Inflamm-Aging Effects of Caloric Restriction}

\section{Introduction}

Much of the aging phenotype, including immunosenescence, can be explained by an imbalance between inflammatory and anti-inflammatory networks, resulting in a chronic low-grade pro-inflammatory status. In 2000, Franceschi [1] called this phenomenon 'inflamm-aging' for the first time, indicating a chronic, progressive increase in the proinflammatory status that occurs as part of the aging process. From this perspective, healthy aging and longevity are likely the results of a lower propensity to mount not only inflammatory responses but also efficient anti-inflammatory networks, leading to a failure to fully neutralize the inflammatory processes consequent to the lifelong antigenic burden and exposure to damaging agents in normal aging [2]. Additionally, inflamm-aging is a determinant of the aging rate and longevity. Data from a case-control study performed in Italian centenarians suggested that a pro-inflammatory genotype did not favor extreme longevity in people with good health but likely favored the onset of age-related diseases such as cardiovascular diseases and Alzheimer's disease, which are among the leading causes of mortality and disability in the elderly [3]. Similarly, Boren noted that increased levels of inflammatory serum markers in the elderly were associated with dementia, Parkinson's disease, atherosclerosis, age-related macular degeneration [4], type 2 diabetes [5], sarcopenia, functional disability and a high mortality risk. Therefore, anti-inflammation might be an effective regulatory countermeasure to delay senescence, and understanding the mechanism of inflamm-aging might be the key to longevity [6].

Toll-like receptor (TLR) 4 [7] is a type of pattern recognition receptor (PRR) that recognizes molecules that are broadly shared by pathogens but distinguishable from host molecules; collectively, these molecules are referred to as pathogen-associated molecular patterns (PAMPs). TLRs, together with the interleukin-1 (IL-1) receptor (IL-1R), form a receptor superfamily known as the 'IL-1R/TLR superfamily'; all of the members of this family have a so-called Toll/IL-1R (TIR) domain in common. TLRs recognize PAMPs and initiate an intracellular kinase cascade to trigger an immediate defense response. In particular, the myeloid differentiation factor 88 (MyD88)-dependent response occurs upon TLR4 dimerization. In this response, ligands such as lipopolysaccharide (LPS) and several heat shock proteins bind and cause changes to the receptor, leading to recruitment of the adaptor protein MyD88, which is a TIR family member. MyD88 then recruits IL-1R-associated kinase (IRAK) 4, IRAK1 and IRAK2. The IRAKs then phosphorylate and activate tumor necrosis factor (TNF) receptor-associated factor 6 (TRAF6), which in turn polyubiquinates TAK1, as well as itself, to facilitate binding to IKK- $\beta$. Upon binding, TAK1 phosphorylates IKK- $\beta$, which

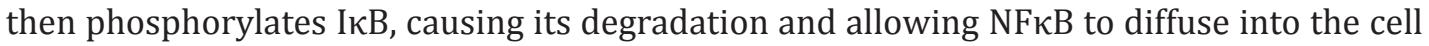
nucleus to activate transcription and the consequent induction of inflammatory cytokines [8]. A schematic diagram of this process is shown in Figure 1.

The TIR domain-containing orphan receptor single immunoglobulin IL-1R-related receptor (SIGIRR), as a member of the TIR superfamily, also plays crucial roles in the immune response. Previous studies have demonstrated that SIGIRR acts as a negative regulator of TLR4 signaling in HepG2 cells, substantially reducing LPS/TLR4-induced nuclear factor kappa-light-chain-enhancer of activated B cells (NF- $\kappa B$ ) activation [9], thereby down-regulating leukocyte adhesion molecules and both pro-inflammatory cytokine and angiogenic factor production. SIGIRR has three components: an Ig domain, a TIR domain and a C-tail. In 2005, Qin [9] demonstrated that only the TIR domain (not including the C-tail part) was necessary for SIGIRR to inhibit TLR4 signaling. SIGIRR specifically attenuates the recruitment of MyD88 adaptors by TLR4 via its intracellular TIR domain, indicating that SIGIRR competitively associates with key signaling proteins such as MyD88. As a result, the total number of adaptor molecules may increase. Therefore, SIGIRR blocks downstream signal transduction and suppresses the intracellular kinase cascade to stop immediate inflammation signal transduction [10].

Caloric restriction (CR) has long been recognized as a modulator of kidney health in both humans and experimental models [11]. In fact, CR can retard the progression of many 
Fig. 1. SIGIRR inhibits the TLR/NF- $\mathrm{KB}$ signaling pathway. SIGIRR acts as a negative regulator of the TLR signaling pathway via its common TIR domain, substantially reducing LPS/TLR4-induced NF- $\mathrm{\kappa B}$ activation and thereby down-regulating downstream signaling molecules and pro-inflammatory cytokines. Therefore, the TLR/NF- $\kappa \mathrm{B}$ signaling pathway is restrained.

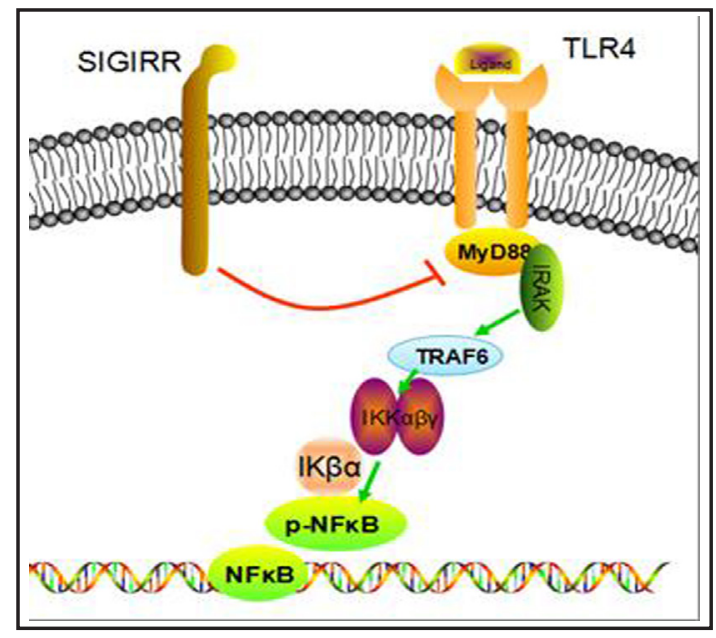

age-associated molecular, physiological, and pathological processes that occur not only in the kidneys [12] but also in the skeletal muscle, heart and brain [13, 14]. Additionally, CR has been shown to effectively reduce chronic progressive nephropathy and ameliorate renal autophagy and oxidative damage in many studies $[15,16]$. In previous studies, CR has also been shown to play a significant role in the anti-inflamm-aging process by decreasing the levels of inflammatory markers in aging tissues. For example, article published in 2014 showed that $\mathrm{CR}$ exerted significant anti-inflammatory effects, preventing age-related increases in NF- $\kappa \mathrm{B}$ transcriptional activity and age-associated pro-inflammatory shifts in cerebromicrovascular endothelial cells [17]. Mustafa Mohammadi also demonstrated that a period of 6 weeks of CR prevented lead-induced inflammation by decreasing the levels of the inflammatory cytokine TNF- $\alpha$ in rat livers [18]. However, thus far, the anti-inflammatory effects of CR have only been superficially examined, and the underlying mechanism has not yet been elucidated. The present study is for the first time to demonstrate the possible regulatory mechanism by which CR induces anti-inflamm-aging and to explore the expression of the upstream and downstream molecules in the TLR4/NF- $\mathrm{KB}$ signaling pathway in this context.

\section{Materials and Methods}

\section{Animals and diets}

Twelve-week-old Fisher 344 (F344) rats were kept under specific pathogen-free conditions: $22 \pm 1^{\circ} \mathrm{C}$, $40 \%$ humidity, a 12:12-h light/dark cycle, and free access to water. All of the rats were fed standard rat chow containing $10.0 \%$ water, $23.0 \%$ crude protein, $51.0 \%$ crude carbohydrate, $5.0 \%$ crude fat, $4.0 \%$ crude fiber, and $7.0 \%$ crude ash, with $3.42 \mathrm{kcal} / \mathrm{g}$ (Keao, Beijing, China). At 16 months of age, the rats were divided into 2 groups: an ad libitum-fed (AL) group and a CR group ( $\mathrm{n}=6$ per group). During the subsequent 8 months, the rats in the CR group were fed an amount of food corresponding to $60 \%$ of that fed to the AL group. Food consumption was measured every 2 weeks, and the results were used to calculate the daily food intake. Vitamins, minerals, and salts were added to the daily food of the diet-restricted animals to ensure that the rats were not nutrient or salt deficient compared with the control animals.

At 24 months old (aged), the AL and CR rats were sacrificed. For this purpose, the rats were anesthetized via an intraperitoneal injection of sodium pentobarbital $(40 \mathrm{mg} / \mathrm{kg})$. Urine was collected in a tube using individual metabolic cages over a 24-hour period before sacrifice, and blood samples were collected from the tail vein to measure metabolic parameters and renal function. To remove the remaining blood, the kidney tissues were perfused with ice-cold, isotonic phosphate-buffered saline (PBS; pH 7.4). A portion of the kidney tissue was immediately fixed in $10 \%$ formalin overnight for histological examination. The remaining tissue was immediately frozen in liquid nitrogen and was stored at $-80^{\circ} \mathrm{C}$ for further use. The rats were maintained in accordance with guidelines for the care and use of laboratory animals, and all of the protocols were approved by the Institutional Animal Care and Use Committee at PLA General Hospital in China. 


\section{Cellular Physiology Cell Physiol Biochem 2015;37:1257-1270 \begin{tabular}{l|l} 
DOI: 10.1159/000430248 & (C) 2015 S. Karger AG, Basel
\end{tabular} www.karger.com/cpb \\ Xu et al.: Anti-Inflamm-Aging Effects of Caloric Restriction}

\section{Biochemical analyses}

The blood samples were centrifuged, and the serum was sent to the Department of Biochemistry at PLA General Hospital to measure total protein (TP), albumin (Alb), blood urea nitrogen (BUN), serum creatinine (SCR), glucose (GLU), triglycerides (TG) and total cholesterol (CHOL). The urine samples were also tested to detect the urinary protein/creatinine ratio.

\section{Renal pathological grading}

The kidneys were processed by two independent investigators. The paraffin-embedded kidney sections were stained with periodic acid-schiff (pas) stain for histological evaluation. Each specimen was then evaluated according to the standards described below $[19,20]$, which classified the histopathology into three types: glomerular lesions, renal interstitial lesions, and vascular lesions. The tubulointerstitial lesion rating included interstitial inflammatory cell infiltration, renal tubular epithelial cell degeneration, renal tubular atrophy and interstitial fibrosis. We used the National Institutes of Health (NIH) Semi-Quantitative Score to measure these indices. In particular, according to the proportion of lesions relative to the renal cortex, we used standard scores of 0 points $(0 \%), 1$ point $(<25 \%), 2$ points $(25-50 \%)$, and 3 points $(>50 \%)$. Active pathological glomerular changes included cell proliferation, leukocyte infiltration, loop necrosis/ karyorrhexis, and cellular crescents, and chronic glomerular renal lesions included glomerular sclerosis and fibrous crescents. According to the percentage of pathological vessels, the measurement criteria were scored as 0 points $(0 \%), 1$ point $(<10 \%), 2$ points $(10-25 \%)$, and 3 points $(>25 \%)$ (Table 1$)$.

\section{Periodic acid-Schiff (PAS) staining}

Kidney slices were fixed in 10\% formalin solution, automatically dehydrated using a series of graded alcohol, embedded in paraffin, sectioned at $4 \mu \mathrm{m}$, subjected to histological staining with PAS, and then examined under a microscope. The investigator was blinded while performing the image analysis, which was based on 20 random glomeruli per rat or 20 random fields per rat at a total magnification of 400x.

\section{Immunofluorescence staining}

Renal tissues were embedded in paraffin. After being deparaffinized, the 4- $\mu$ m-thick sections were subjected to antigen retrieval by microwaving or autoclaving for 10 or $15 \mathrm{~min}$ in $10 \mathrm{mM}$ sodium citrate buffer (pH 6.0) and were then stained with rabbit polyclonal anti-MyD88 antibody (Abcam) at 1:100, rabbit polyclonal phospho-NF- $\mathrm{KB}$ p65 antibody (Cell Signaling Technology) at 1:100 and rabbit polyclonal anti-SIGIRR antibody (Abcam) at 1:100. Next, the sections were sequentially incubated with rhodamine-

Table 1. Renal pathological grading from $0-3$

\begin{tabular}{|c|c|c|c|c|}
\hline SCORE & 0 & 1 & 2 & 3 \\
\hline \multicolumn{5}{|l|}{ Glomerular Lesions } \\
\hline Cell Proliferation & - & Mild & Medium & Severe \\
\hline Leukocyte Infiltration & 0 & $<25 \%$ & $25-50 \%$ & $>50 \%$ \\
\hline Loop Necrosis/Karyorrhexis x 2 & 0 & $<25 \%$ & $25-50 \%$ & $>50 \%$ \\
\hline Cellular Crescents x 2 & 0 & $<25 \%$ & $25-50 \%$ & $>50 \%$ \\
\hline Glomerulosclerosis & 0 & $<25 \%$ & $25-50 \%$ & $>50 \%$ \\
\hline Fibrous Crescent & 0 & $<25 \%$ & $25-50 \%$ & $>50 \%$ \\
\hline \multicolumn{5}{|l|}{ Renal Interstitial Lesions } \\
\hline $\begin{array}{l}\text { Interstitial Inflammatory Cell } \\
\text { Infiltration }\end{array}$ & - & Mild & Medium & Severe \\
\hline $\begin{array}{l}\text { Renal Tubular Epithelial Cell } \\
\text { Degeneration }\end{array}$ & - & Mild & Medium & Severe \\
\hline Renal Tubular Atrophy & - & Mild & Medium & Severe \\
\hline Renal Interstitial Fibrosis & - & Mild & Medium & Severe \\
\hline Protein Casts & - & $<10 \%$ & $10-25 \%$ & $>25 \%$ \\
\hline
\end{tabular}




\section{Cellular Physiology Cell Physiol Biochem 2015;37:1257-1270 \begin{tabular}{l|l|l}
\hline DOI: 10.1159/000430248 & (C) 2015 S. Karger AG, Basel
\end{tabular} \begin{tabular}{l|l|} 
and Biochemistry Published online: October 05, 2015 & www.karger.com/cpb \\
\cline { 1 - 2 } &
\end{tabular} \\ Xu et al.: Anti-Inflamm-Aging Effects of Caloric Restriction}

conjugated AffiniPure goat anti-rabbit IgG followed by fluorescein-conjugated AffiniPure goat anti-mouse IgG (Jackson ImmunoResearch Laboratories, West Grove, PA, USA). The results were analyzed under an Olympus laser scanning confocal microscope (IX81), and the investigator was blinded while performing the image analysis. Quantitative analysis of fluorescence-marked areas in 20 glomeruli per rat was performed using Image-Pro software at a total magnification of $20 \mathrm{x}$.

\section{Immunohistochemistry}

The kidneys were fixed in $10 \%$ formaldehyde overnight at $4{ }^{\circ} \mathrm{C}$ and then embedded in paraffin following standard procedures. After being deparaffinized, the 4- $\mu \mathrm{m}$-thick sections were subjected to antigen retrieval by microwaving or autoclaving for 10 or $15 \mathrm{~min}$ in $10 \mathrm{mM}$ sodium citrate buffer (pH 6.0). After $10 \mathrm{~min}, 3 \%$ hydrogen peroxide was used to block endogenous peroxidase activity. After washing with PBS, the sections were incubated with $1.5 \%$ normal goat serum for $30 \mathrm{~min}$, followed by incubation with rabbit polyclonal anti-SIGIRR antibody (Abcam) overnight at $4^{\circ} \mathrm{C}$. Following three washes with PBS, the samples were incubated with biotin-conjugated goat anti-rabbit IgG (Invitrogen Corporation, CA, USA) for $30 \mathrm{~min}$ at room temperature. After another wash with PBS, the sections were incubated with streptavidinconjugated peroxidase (Invitrogen Corporation, CA, USA) for $30 \mathrm{~min}$ at room temperature and were then incubated with diaminobenzidine (DAB; Invitrogen Corporation, CA, USA), followed by observation under a microscope.

\section{Western blot analysis}

Kidney tissues were lysed with RIPA buffer (50 mM Tris-Cl [pH 7.6], $150 \mathrm{mM} \mathrm{NaCl,} \mathrm{1 \%} \mathrm{NP-40,} \mathrm{0.1 \%}$ SDS, $0.5 \%$ deoxycholic acid, $1 \mathrm{mg} / \mathrm{mL}$ leupeptin, $1 \mathrm{mg} / \mathrm{mL}$ aprotinin, and $0.5 \mathrm{mM}$ phenylmethylsulfonyl fluoride) for $30 \mathrm{~min}$ on ice before centrifugation at $12000 \mathrm{rpm}$ for $30 \mathrm{~min}$ at $4^{\circ} \mathrm{C}$ to separate the cellular proteins into the supernatant. The protein concentration was measured with a Pierce BCA assay kit (Lot \#JK126465; Thermo Fisher Scientific, Rockford, IL, USA). A total of 50-100 mg protein from each sample was resolved by $8-12 \%$ SDS-PAGE and then transferred to nitrocellulose (NC) membranes, blocked with $10 \%$ casein for $30 \mathrm{~min}$ at room temperature, and probed with the following primary antibodies at $4{ }^{\circ} \mathrm{C}$ overnight: rabbit polyclonal anti-HMGB1 antibody (Novus Biologicals, 1:1000), mouse monoclonal antiTLR4 antibody (Abcam, 1:500), rabbit polyclonal anti-MyD88 antibody (Abcam, 1:1000), anti-TRAF6 antibody (Abcam 1:2000), rabbit polyclonal phospho-NF- $\kappa$ B p65 antibody (Cell Signaling Technology, 1:1000), rabbit polyclonal anti-SIGIRR antibody (Abcam, 1:2000), rabbit polyclonal anti-IRAK4 antibody (Novus Biologicals, 1:1000), mouse monoclonal phospho-IK $\beta \alpha$ antibody (Cell Signaling Technology, 1:500), and mouse monoclonal beta-actin antibody (Sigma-Aldrich, 1:5000). The blots were subsequently incubated with horseradish peroxidase-conjugated anti-rabbit or anti-mouse IgG secondary antibodies (Santa Cruz Biotechnology) at 1:1000-1:5000. Immunoreactive bands were visualized by enhanced chemiluminescence, and densitometry was performed using Image software version 1.48 (NIH, Bethesda, MD, USA).

\section{Statistical analysis}

The results are reported as the mean \pm standard deviation (SD) using SPSS software, version 17.0 (SPSS, Chicago, IL, USA). Statistical significance was accepted at a level of $\mathrm{p}<0.05$. The $\mathrm{t}$ test was used to measure the variance of the data.

\section{Results}

Effects of CR on metabolic parameters in aged rats

The body weight of the rats in the CR group gradually decreased to $322.3 \pm 12.8 \mathrm{~g}$, which was significantly $(\mathrm{p}<0.001)$ lower than the weight of the rats in the AL control group $(499.5 \pm 24.2 \mathrm{~g})$. Furthermore, the kidney weight significantly differed between these two groups ( $2.86 \pm 0.05$ vs. $2.22 \pm 0.11$, respectively). Differences in metabolic parameters were also observed between the two groups of F344 rats: by the end of the experiment, the GLU and BUN levels, TP, Alb, TG, and urine protein/urine creatinine ratio were significantly lower in the rats in the CR group than in the rats in the AL control group. However, SCR and CHOL were not significantly different between the two groups over the 8-month experimental period. The details are shown in Table 2 . 


\section{Cellular Physiology Cell Physiol Biochem 2015;37:1257-1270 \begin{tabular}{l|l|l}
\hline DOI: 10.1159/000430248 & (C) 2015 S. Karger AG, Basel
\end{tabular} \begin{tabular}{l|l|} 
and Biochemistry Published online: October 05, 2015 & www.karger.com/cpb \\
\cline { 1 - 2 } &
\end{tabular}

Table 2. Metabolic parameters in the CR and AL groups. The data are presented as the mean \pm SD $(n=5-6) . p<0.05$ for AL feeding vs. CR

\begin{tabular}{lll}
\hline & AL Group & CR Group \\
\hline Body weight (g) & $499.5 \pm 24.2$ & $322.3 \pm 12.8^{* * * *}$ \\
Kidney weight (g/L) & $2.86 \pm 0.05$ & $2.22 \pm 0.11^{* *}$ \\
Total protein (g/L) & $76.27 \pm 1.39$ & $65.00 \pm 0.72^{* * *}$ \\
Albumin (g/L) & $50.50 \pm 3.14$ & $39.90 \pm 3.64^{* * *}$ \\
Glucose (mmol/L) & $8.47 \pm 0.61$ & $5.42 \pm 3.64^{*}$ \\
Serum urea nitrogen (mmol/L) & $5.72 \pm 0.75$ & $5.27 \pm 0.40^{*}$ \\
Creatinine (mmol/L) & $30.27 \pm 3.25$ & $28.00 \pm 2.40$ \\
Cholesterol (mmol/L) & $3.68 \pm 0.40$ & $2.97 \pm 2.40$ \\
Triglycerides (mmol/L) & $1.01 \pm 0.07$ & $0.42 \pm 0.09^{* * *}$ \\
Urinary albumin/urine creatinine & $533.33 \pm 159.01$ & $79.91 \pm 34^{*}$ \\
\hline
\end{tabular}

Renal pathological grading measurement and representative picture

Renal pathological grading was based on past studies [19, 20]. Pathological scoring of the renal sections revealed evidence of tissue damage on a scale from 0-3 points; nonetheless, no pathological evidence of damage graded as 3 points was observed. Among the glomerular changes, no fibrous crescents were found in either group. However, cell proliferation, leukocyte infiltration, loop necrosis, and glomerulosclerosis were all significantly reduced $(\mathrm{p}<0.05)$ in the rats in the CR group compared with the rats in the AL group. Furthermore, interstitial inflammatory cell infiltration, renal tubular epithelial cell degeneration, renal tubular atrophy and interstitial fibrosis were evaluated; all of these indices were significantly decreased in the CR group compared with the AL group. Representative pictures based on the evaluation criteria and pathological scores are shown in Figure 2.

SIGIRR expression decreases with increasing age, and CR can cause SIGIRR overexpression

The immunoregulatory function of SIGIRR is derived from its ability to constrain the inflammatory consequences of TLR4 activation. SIGIRR overexpression can specifically block the TLR/NF- $\kappa \mathrm{B}$ pathway, thereby suppressing active inflammation. Here, to assess the role of SIGIRR in mediating the response to TLR4 activation during the aging process, western blotting was conducted to compare SIGIRR expression between young ( 3 months old) and old (24 months old) rats. In the young rats, SIGIRR expression was significantly higher than in the old rats, indicating that SIGIRR expression decreases during the aging process. A similar phenomenon was observed between the CR group and the AL control group, with significantly higher SIGIRR expression in the CR group. Immunofluorescence revealed that SIGIRR was mainly expressed on the lateral membrane of renal tubular epithelial cells, with little glomerular expression. Moreover, the AL group showed high fluorescence intensity compared with the CR group. These findings were in accordance with the immunohistochemical results. Additionally, the intensity of SIGIRR expression was higher in the CR group during immunohistochemistry (see Fig. 3).

\section{Effect of CR on the TLR4 molecule}

TLR4 is a TLR that detects LPS from Gram-negative bacteria and is thus important in the activation of the innate immune system. In the present study, western blotting showed that TLR4 expression was increased in the CR rats compared with the AL-fed control rats. Although increased TLR4 expression is typically thought of as equivalent to the activation of inflammation in theory, the limited studies that have been published on this topic to date have yielded discrepant results. For example, certain studies have reported no significant difference between CR and AL groups [21], whereas in the present study, the expression of TLR4 was significantly higher in the CR group than in the AL group. As it was possible that 

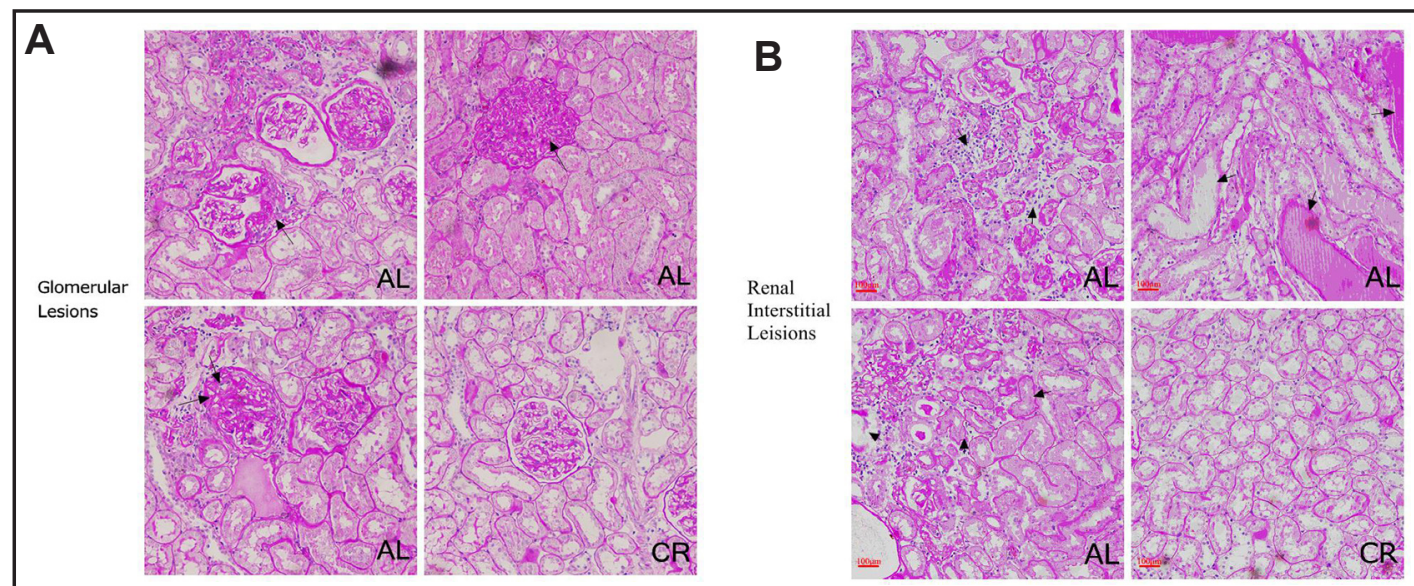

Fig. 2. Representative pictures and histology scores of glomerular and tubulointerstitial lesions based on standard procedures (compared with the AL group, * $\mathrm{p}<0.05)$. A. The black arrow on the upper left shows a fibrous crescent in the glomerular lesions. The black arrow on the upper right shows cell proliferation in the glomerular lesions. The black arrows on the lower left show glomerulosclerosis in the glomerular lesions. Figure on the lower right show that CR can alleviate glomerular lesions. B. The black arrows on the upper left show interstitial inflammatory cell infiltration in the tubulointerstitial lesions. The black arrows on the upper right show protein casts in the tubulointerstitial lesions. The

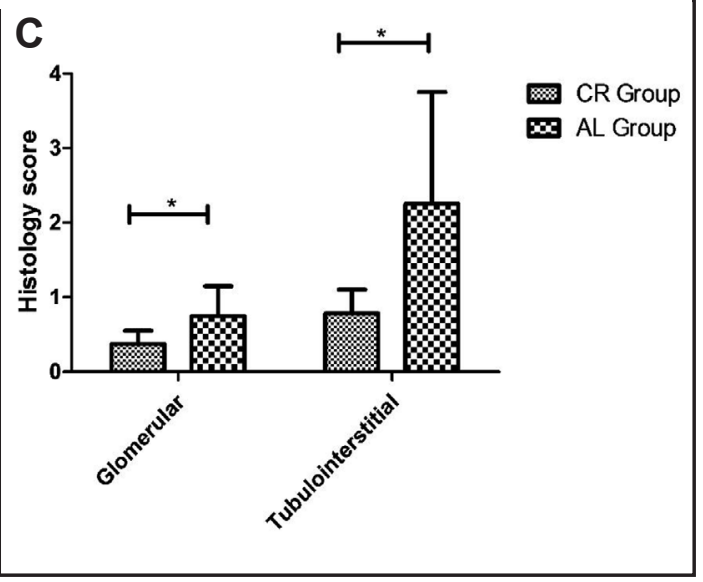
black arrows on the lower left show renal tubular atrophy and renal tubular epithelial cell degeneration in the tubulointerstitial lesions. Figure on the lower right show that CR can alleviate tubulointerstitial lesions. C. histology scores based on standard procedures were significantly decreased in the CR group compared with the AL group.

Fig. 3. Changes in TLR4 expression changes in TLR4. Western blotting shows high expression in the CR group compared with the AL group (compared with the AL group, ${ }^{*} \mathrm{p}<0.05, \mathrm{n}=12$ per group).

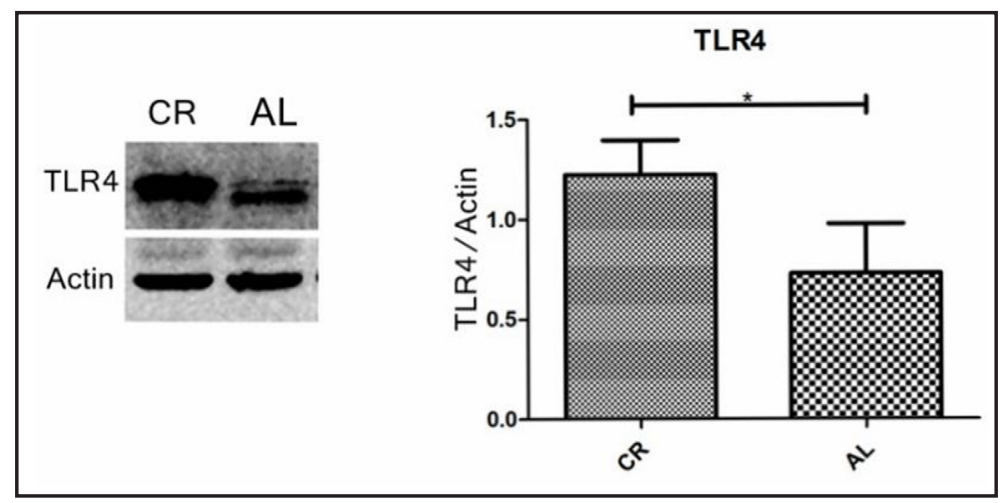

the competitive effect of the overexpression of SIGIRR led to a compensatory increase in TLR4 expression, we sought to confirm this possibility (see Fig. 4).

SIGIRR overexpression can decrease the expression of the adaptor molecules MyD88, IRAK4 and TRAF6

The adaptor proteins and kinases that mediate TLR signaling have also been studied. When activated, TLRs recruit adaptor molecules within the cytoplasm of cells to propagate the signal. The MyD88, IRAK4 and TRAF6 adaptor molecules are known to be 
Fig. 4. Changes in SIGIRR expression. A. Western blotting shows high expression in young rats compared with old rats. B. Western blotting shows high expression in the CR group compared with the AL group. C. Immunofluorescence results show that positive granular staining for SIGIRR was generally located on the lateral membrane of renal tubular epithelial cells, whereas there was markedly little staining in the glomerulus (indicated by white arrows). Moreover, the AL group showed high fluorescence intensity compared with the CR group. D. Immunohistochemical staining shows similar results, with positive granular staining for SIGIRR generally located on the lateral membrane of renal tubular epithelial cells (indicated by black arrows) and a higher intensity of SIGIRR expression in the CR group (compared with the AL group, * $\mathrm{p}<0.05$, ${ }^{*} \mathrm{p}<0.01, \quad * * * \mathrm{p}<0.001$, $\mathrm{n}=6$ per group).

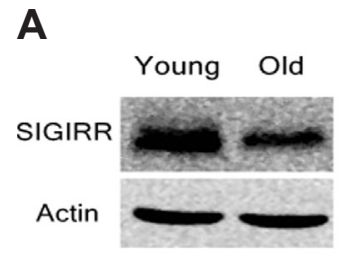

B
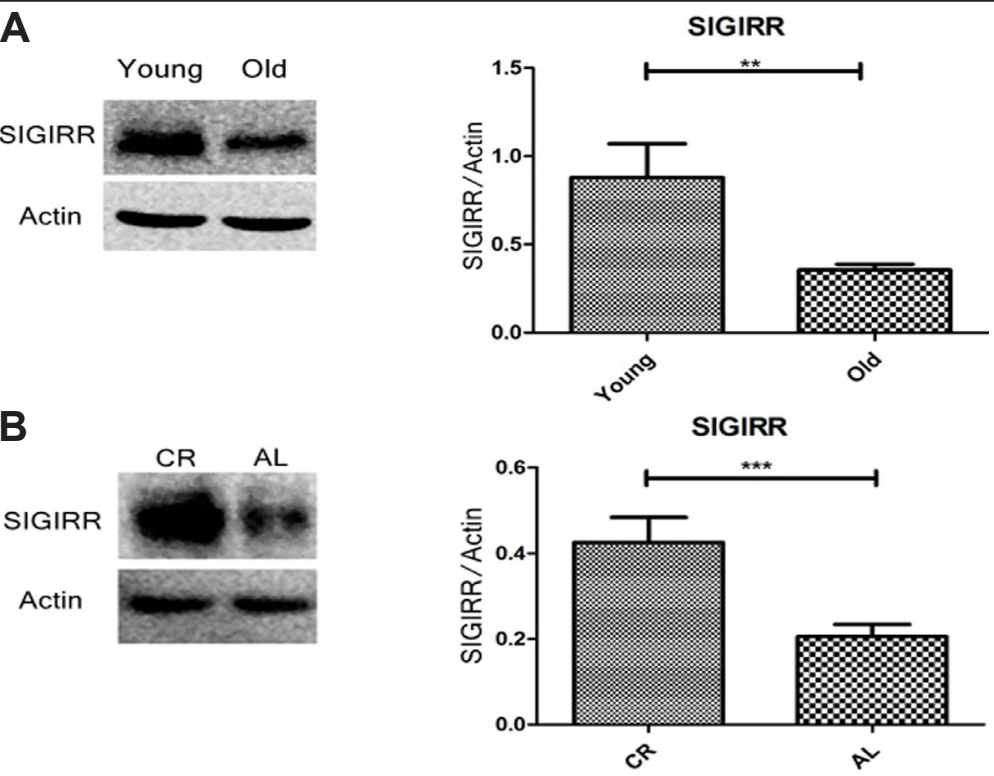

C
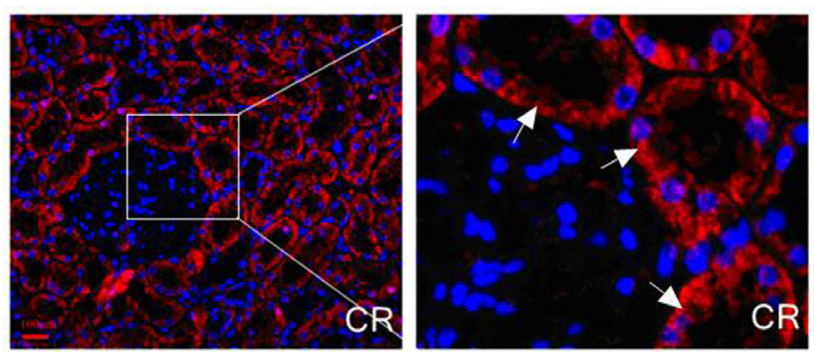

D
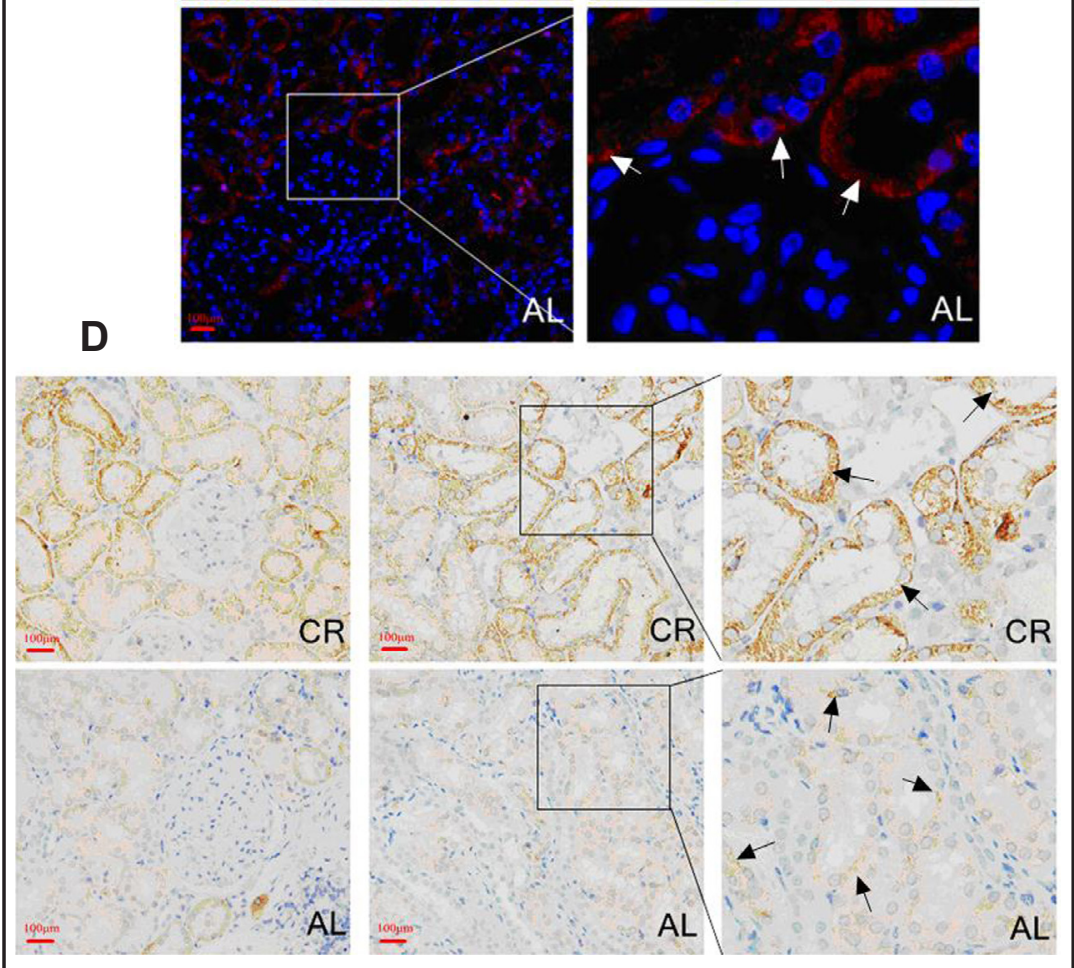

involved in the TLR4/NF- $\mathrm{B}$ signaling pathway in particular. A previous study [22] used a co-immunoprecipitation assay to demonstrate that SIGIRR overexpression had a greater ability to inhibit downstream inflammation upon binding to those adaptor molecules 
Fig. 5. Changes in MyD88, IRAK4 and TRAF6 expression. A. Western blotting shows low MyD88 expression in the CR group compared with the AL group. B. Western blotting shows that IRAK4 expression was decreased in CR rats compared with the AL control rats. C. Western blotting shows that TRAF6 expression was decreased in CR rats compared with the AL control rats. D. Immunofluorescence results show that positive granular staining for MyD88 was generally located in the basement membrane of renal tubular epithelial cells (indicated by white arrows) and that the CR group exhibited lower fluorescence intensity compared with the AL group (compared with the AL group, ${ }^{*} \mathrm{p}<0.05$, $* * \mathrm{p}<0.01, \mathrm{n}=6$ per group).

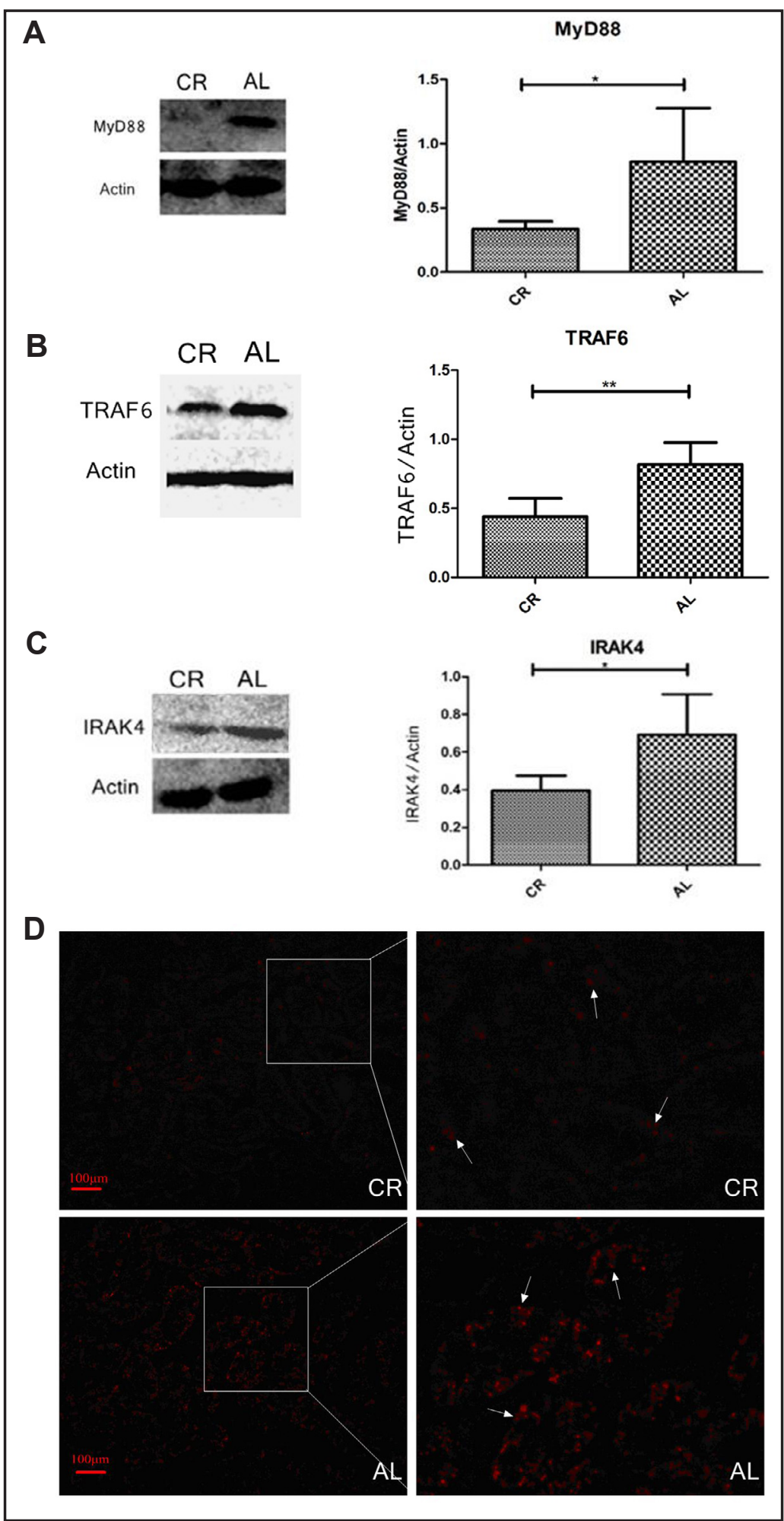

via its common TIR domain. In the present study, MyD88 expression was significantly lower in the CR group. IRAK4 and TRAF6, which are the other adaptor molecules that are downstream of MyD88, exhibited significantly lower expression in the CR group compared with the AL group, which was in agreement with the results of related studies. Based on immunofluorescence analysis, MyD88 was mainly located in the basement membrane of 
Fig. 6. Changes in phospho-IK $\beta \alpha$ and phospho-NFKB expression. A. Western blotting shows that phospho-IK $\beta \alpha$ was highly expressed in the AL group compared with the CR group. B. Western blotting shows that phospho-NFкB expression was decreased in $\mathrm{CR}$ rats compared with the AL control rats. C. Immunofluorescence results show that positive granular staining for phospho-NFkB was generally located in the nucleus of glomerular mesangial cells (indicated by white arrows) and that the CR group exhibited lower fluorescence intensity compared with the AL group (compared with the AL group, * $\mathrm{p}<0.05$, $\mathrm{n}=6$ per group).

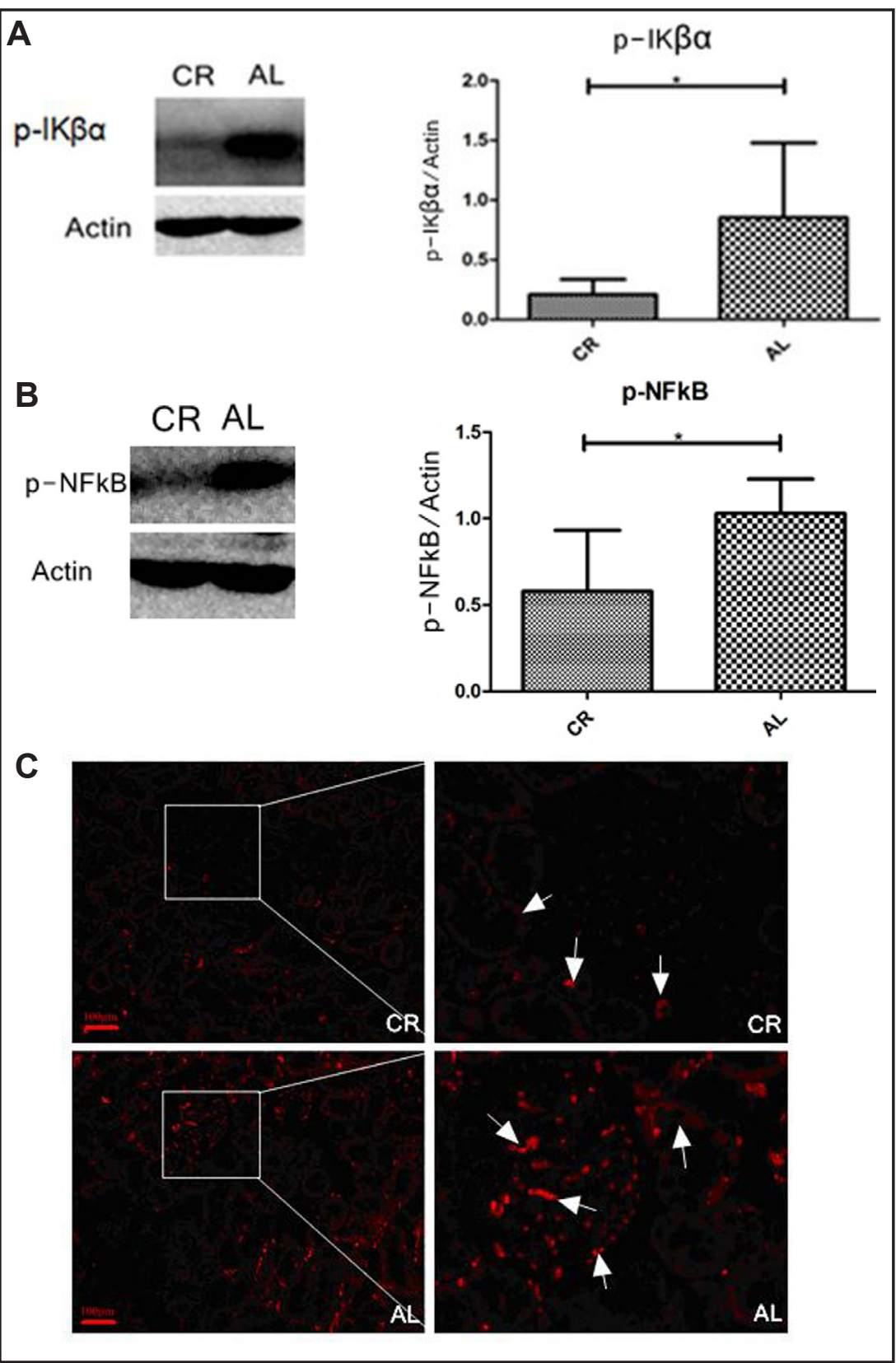

renal tubular epithelial cells (indicated by white arrows), and the CR group showed lower fluorescence intensity compared with the AL group (Fig. 5).

\section{Inflammatory marker expression decreases during $C R$}

NF- $\kappa B$ is a key transcription factor that activates inflammatory gene expression in response to stimuli and that regulates the intracellular expression of pro-inflammatory cytokines. Phospho-I $\mathrm{KB} \alpha$ is an important marker that demonstrates the activation of NF$\kappa \mathrm{B}$. Before its phosphorylation, $\mathrm{I} \kappa \mathrm{B} \alpha$, as the most powerful inhibitor of NF- $\kappa \mathrm{B}$, can mask the nuclear localization signals (NLSs) of NF- $\kappa B$ proteins, keeping them sequestered in an inactive state in the cytoplasm. I $\mathrm{B} \alpha$ is phosphorylated after receiving the activating signal of upstream signaling factors. I $\kappa \mathrm{B} \alpha$ phosphorylation is followed by its polyubiquitination and degradation by the proteasome and the release of NF- $\kappa B$. The liberated NF- $\kappa B$ then translocates to the nucleus and triggers a serial cascade of pathophysiological reactions, thereby inducing the release of certain inflammatory intermediates. NF- $\kappa B$ p65 is the most 


\section{Cellular Physiology Cell Physiol Biochem 2015;37:1257-1270 \begin{tabular}{l|l} 
and Biochemistry & $\begin{array}{l}\text { DOI: 10.1159/000430248 } \\
\text { Published online: October 05, } 2015\end{array}$ \\
\hline
\end{tabular} \\ Xu et al.: Anti-Inflamm-Aging Effects of Caloric Restriction}

important subunit of NF- $\kappa \mathrm{B}$, playing a key role in the transcriptional activation of the protein. Phospho-NF- $\mathrm{BB}$ p65 has transcriptional activity, and its expression is also correlated with transcriptional activity. Therefore, in the current study, western blotting was used to detect

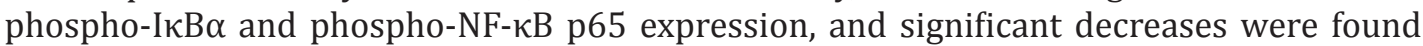
in the CR group compared with the AL group. Additionally, immunofluorescence analysis showed that positive granular staining for phospho-NFKB is generally located in the nucleus of glomerular mesangial cells (indicated by white arrows) and that the CR group exhibited lower fluorescence intensity compared with the AL group (Fig. 6).

\section{Discussion}

Chronic inflammation is now accepted as a major underlying risk factor for many agerelated diseases, such as atherosclerosis, diabetes, osteoporosis, vascular diseases, metabolic syndrome, obesity and cancer [23]. However, only a limited number of studies have seriously considered the role of inflammation in the aging process. In 2006, Chung [24] demonstrated that chronic, low-grade inflammation was a possible converging process linked to normal aging and to age-related diseases. Several new findings were also recently reported, providing us with new insights into age-related physiological changes and the pathogenesis of many age-related diseases in the aging process and opening up new avenues for exploration of the interrelationships between aging and age-related molecular mechanisms. NF- $\kappa B$ is a key transcription factor that regulates the intracellular expression of various pro-inflammatory cytokines. Salminen A [25] reported that NF- $\kappa B$ signaling was the molecular culprit in inflamm-aging because it activates the innate immune system during aging. The NF- $\kappa B$ system is located at a nodal point, linking pathogenic assault signals and cellular danger signals and then organizing cellular resistance. Recent studies have revealed that SIRT1 and FoxO, which are the key regulators of aging in budding yeast and Caenorhabditis elegans models and which are known as longevity genes, regulate the NF- $\kappa B$ signaling efficiency and the inflammatory response level [26]. Many researchers now accept CR as the only established anti-aging experimental paradigm. Consequently, much attention has been paid to the antiinflammatory potential of CR. Indeed, CR attenuates age-related increases in the systemic levels of several pro-inflammatory mediators $[27,28]$, but the possible mechanisms of this effect remain unclear. In the present study, for the first time, we extensively examined the effects of CR, which might be used to mitigate age-related inflammation, on inflammation in the aging process. CR might represent a promising approach in humans, and especially the elderly.

In the present study, for weight gain analyses, we monitored the weights of the animals weekly. After 8 months, the rats in the CR group showed 35\% less weight gain than the rats in the AL group did; this difference was statistically significant. These results were in agreement with previous data published by our research group, suggesting that the CR model was successfully established. At the end of the experimental period, biochemical serum analyses were performed to assess the nutritional and metabolic statuses of the rats fed an AL or CR diet. No significant differences were observed between the AL-fed and the CR animals in terms of SCR and CHOL. However, CR caused a significant reduction in certain serum parameters, including GLU and BUN levels, TP, Alb, TG, and urine protein/urine creatinine ratio, possibly indicating that CR has an underlying effect on chronic kidney diseases. These findings were consistent with previous results [29, 30].

Among renal morphological changes, we observed inflammatory cell infiltration, shrinkage of Bowman's capsule and mesangial area expansion. However, these pathological changes were alleviated in the CR group. Studies of renal morphological changes in chronic kidney disease have particularly emphasized events observed in the advanced lesions of glomerulosclerosis and in tubulointerstitial lesions. In the present study, 40\% CR for 8 months could alleviate both glomerulosclerosis and tubulointerstitial lesions; however, the possible mechanisms of the inflammatory effects on the age-associated renal structure 
and functional remodeling remain unclear. In 2009, Mei C [31] demonstrated that the inflammatory cytokine TGF- $\beta$ might transform fibroblasts into myofibroblasts, leading to abundant collagen synthesis as well as to glomerular sclerosis and interstitial fibrosis. TGF- $\beta$ also induces remarkable proliferation of glomerular mesangial cells and interstitial fibroblasts through the c-abl and PAK2 pathways. Recently, Jiang further showed that agerelated renal disease is accompanied by increased lipid accumulation, which is regulated by increased SREBP expression [32].

Recent studies have found that SIGIRR is closely associated with kidney disease. The basic mechanism involves risk recognition via SIGIRR signaling in response to inflammatory/ immune damage. To date, however, only a limited number of studies have addressed this issue in the kidneys. The present study fills this gap. In this study, SIGIRR was highly expressed in renal tubular epithelial cells during the CR intervention in vivo. Additionally, previous in vitro studies have demonstrated that intrarenal antigen-presenting cells and tubular epithelial cells in culture dominantly expressed SIGIRR [33]. A lack of TIR8/SIGIRR is associated with improved renal chemokine pathway signaling upon stimulus exposure. Furthermore, in his studies in 2012, Leemans JC suggested that SIGIRR is an important negative regulator of an LPS-mediated inflammatory response in tubular epithelial cells and dampens an effective antibacterial host response during pyelonephritis [34]. Additionally, SIGIRR plays a crucial role in renal fibrosis [10]. SIGIRR can also inhibit TLR-induced cytokine and chemokine expression, and its deficiency aggravates postobstructive renal fibrosis [10].

TLR4 belongs to the TLR/IL-1R superfamily, which is defined by a common TIR domain in the cytoplasm. TLR4, which is located in the cell membrane, recognizes PAMPs and can initiate an intracellular kinase cascade to trigger NF- $\kappa B$ signaling. SIGIRR, another member of the TLR/IL-1R superfamily, acts as a negative regulator of MyD88-dependent TLR signaling and attenuates MyD88 adaptor recruitment to receptors via its common intracellular TIR domain. Thus, SIGIRR is a highly important molecule to target in therapy for the inflammation-related diseases caused by TLR4. In the current study, for the first time, we explored the effect of SIGIRR on TLR/NF- $\kappa B$ signaling in a CR model. We demonstrated that CR could enhance SIGIRR expression. Due to the competitive binding of SIGIRR with MyD88 via its TIR domain, CR could also inhibit substantial NF- $\kappa B$ activation by TLR4.

In summary, our study demonstrated that 8-month CR in aged rats could decrease inflammatory activity in the aged rats' kidneys and could block TLR4/NF- $\mathrm{BB}$ signaling via SIGIRR overexpression. As shown in previous studies, SIRT1, AMPK and mTOR are upstream regulatory molecules that are associated with the slowing of aging-related kidney damage by CR in aged rats [35]. CR also deactivates the mTOR pathway by activating AMPK and SIRT1 $[36,37]$. Therefore, SIGIRR overexpression is likely regulated by one of these upstream molecules (AMPK, SIRT1 or mTOR). However, this hypothesis requires further study to be confirmed. Our results could be useful in understanding how CR prevents aberrations in kidney structure and function during aging and how CR works during the onset and development of kidney aging and kidney disease, as well as in exploring new therapeutic interventions that could delay inflamm-aging in renal disease. Based on these data, CR supplementation could be considered as a potential nutritional paradigm and a suppressive measure to slow renal inflamm-aging and age-related deterioration.

\section{Disclosure Statement}

The authors have no conflict of interest to declare, and none of the studies included in our analyses had a conflict of interest. This project was funded by the National Key Basic Research Program of China (973 Program), (2013CB530800), National High Technology Research and Development Program of China(863 program)(2012AA02A512), and the NSFC (81070267, 81171645). 


\section{Cellular Physiology Cell Physiol Biochem 2015;37:1257-1270 \begin{tabular}{l|l} 
and Biochemistry Published online: October 05, 2015 & $\begin{array}{l}\text { C 2015 S. Karger AG, Basel } \\
\text { www.karger.com/cpb }\end{array}$ \\
\hline
\end{tabular} \\ Xu et al.: Anti-Inflamm-Aging Effects of Caloric Restriction}

\section{References}

1 Franceschi C, Bonafe M, Valensin S, Olivieri F, De Luca M, Ottaviani E, De Benedictis G: Inflamm-aging. An evolutionary perspective on immunosenescence. Ann N Y Acad Sci 2000;908:244-254.

2 Franceschi C, Valensin S, Bonafe M, Paolisso G, Yashin AI, Monti D, De Benedictis G: The network and the remodeling theories of aging: Historical background and new perspectives. Exp Gerontol 2000;35:879-896.

3 Candore G, Caruso C, Colonna-Romano G: Inflammation, genetic background and longevity. Biogerontology 2010;11:565-573.

4 Boren E, Gershwin ME: Inflamm-aging: Autoimmunity, and the immune-risk phenotype. Autoimmun Rev 2004;3:401-406.

5 Franceschi C, Valensin S, Lescai F, Olivieri F, Licastro F, Grimaldi LM, Monti D, De Benedictis G, Bonafe M: Neuroinflammation and the genetics of alzheimer's disease: The search for a pro-inflammatory phenotype. Aging 2001;13:163-170.

6 De Martinis M, Franceschi C, Monti D, Ginaldi L: Inflamm-ageing and lifelong antigenic load as major determinants of ageing rate and longevity. FEBS Lett 2005;579:2035-2039.

7 Rock FL, Hardiman G, Timans JC, Kastelein RA, Bazan JF: A family of human receptors structurally related to drosophila toll. Proc Natl Acad Sci USA 1998;95:588-593.

8 Kawai T, Akira S: The role of pattern-recognition receptors in innate immunity: Update on toll-like receptors. Nat Immunol 2010;11:373-384.

9 Qin J, Qian Y, Yao J, Grace C, Li X: Sigirr inhibits interleukin-1 receptor- and toll-like receptor 4-mediated signaling through different mechanisms. J Biol Chem 2005;280:25233-25241.

10 Skuginna V, Lech M, Allam R, Ryu M, Clauss S, Susanti HE, Rommele C, Garlanda C, Mantovani A, Anders HJ: Toll-like receptor signaling and sigirr in renal fibrosis upon unilateral ureteral obstruction. PLoS One 2011;6:e19204.

11 Anderson S, Brenner BM: Effects of aging on the renal glomerulus. Am J Med 1986;80:435-442.

12 Jiang T, Liebman SE, Lucia MS, Phillips CL, Levi M: Calorie restriction modulates renal expression of sterol regulatory element binding proteins, lipid accumulation, and age-related renal disease. J Am Soc Nephrol 2005;16:2385-2394.

13 McKiernan SH, Bua E, McGorray J, Aiken J: Early-onset calorie restriction conserves fiber number in aging rat skeletal muscle. FASEB J 2004;18:580-581.

14 Nicoletti VG, Marino VM, Cuppari C, Licciardello D, Patti D, Purrello VS, Stella AM: Effect of antioxidant diets on mitochondrial gene expression in rat brain during aging. Neurochem Res 2005;30:737-752.

15 Ning YC, Cai GY, Zhuo L, Gao JJ, Dong D, Cui S, Feng Z, Shi SZ, Bai XY, Sun XF, Chen XM: Short-term calorie restriction protects against renal senescence of aged rats by increasing autophagic activity and reducing oxidative damage. Mech Ageing Dev 2013;134:570-579.

16 Cui J, Shi S, Sun X, Cai G, Cui S, Hong Q Chen X, Bai XY: Mitochondrial autophagy involving renal injury and aging is modulated by caloric intake in aged rat kidneys. PLoS One 2013;8:e69720.

17 Csiszar A, Gautam T, Sosnowska D, Tarantini S, Banki E, Tucsek Z, Toth P, Losonczy G, Koller A, Reglodi D, Giles CB, Wren JD, Sonntag WE, Ungvari Z: Caloric restriction confers persistent anti-oxidative, pro-angiogenic, and anti-inflammatory effects and promotes anti-aging mirna expression profile in cerebromicrovascular endothelial cells of aged rats. Am J Physiol Heart Circ Physiol 2014;307:H292-306.

18 Mohammadi M, Ghaznavi R, Keyhanmanesh R, Sadeghipour HR, Naderi R, Mohammadi H: Caloric restriction prevents lead-induced oxidative stress and inflammation in rat liver. ScientificWorldJournal 2014;2014:821524.

19 Debelle FD, Nortier JL, De Prez EG, Garbar CH, Vienne AR, Salmon IJ, Deschodt-Lanckman MM, Vanherweghem JL: Aristolochic acids induce chronic renal failure with interstitial fibrosis in salt-depleted rats. J Am Soc Nephrol 2002;13:431-436.

20 Bazzi C: A modern approach to selectivity of proteinuria and tubulointerstitial damage in nephrotic syndrome. Kidney Int 2000;58:1732-1741.

21 Horrillo D, Sierra J, Arribas C, Carrascosa JM, Lauzurica N, Ros M: Age-associated development of inflammation in wistar rats: Effects of caloric restriction. Arch Physiol Biochem 2011;117:140-150.

22 Gong J, Wei T, Stark RW, Jamitzky F, Heckl WM, Anders HJ, Lech M, Rossle SC: Inhibition of toll-like receptors tlr4 and 7 signaling pathways by sigirr: A computational approach. J Struct Biol 2010;169:323-330. 


\section{Cellular Physiology Cell Physiol Biochem 2015;37:1257-1270

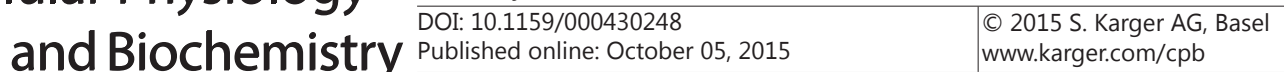 \\ Xu et al.: Anti-Inflamm-Aging Effects of Caloric Restriction}

23 Yu BP, Chung HY: Adaptive mechanisms to oxidative stress during aging. Mech Ageing Dev 2006;127:436443.

24 Chung HY, Sung B, Jung KJ, Zou Y, Yu BP: The molecular inflammatory process in aging. Antioxid Redox Signal 2006;8:572-581.

25 Salminen A, Huuskonen J, Ojala J, Kauppinen A, Kaarniranta K, Suuronen T: Activation of innate immunity system during aging: Nf-kb signaling is the molecular culprit of inflamm-aging. Ageing Res Rev 2008;7:83105.

26 Cohen HY, Miller C, Bitterman KJ, Wall NR, Hekking B, Kessler B, Howitz KT, Gorospe M, de Cabo R, Sinclair DA: Calorie restriction promotes mammalian cell survival by inducing the sirt1 deacetylase. Science 2004;305:390-392.

27 Ershler WB, Keller ET: Age-associated increased interleukin-6 gene expression, late-life diseases, and frailty. Annu Rev Med 2000;51:245-270.

28 Krabbe KS, Pedersen M, Bruunsgaard H: Inflammatory mediators in the elderly. Exp Gerontol 2004;39:687-699.

29 Mark DA, Alonso DR, Quimby F, Thaler HT, Kim YT, Fernandes G, Good RA, Weksler ME: Effects of nutrition on disease and life span. I. Immune responses, cardiovascular pathology, and life span in mrl mice. Am J Pathol 1984;117:110-124.

30 Mark DA, Alonso DR, Tack-Goldman K, Thaler HT, Tremoli E, Weksler BB, Weksler ME: Effects of nutrition of disease and life span. Ii. Vascular disease, serum cholesterol, serum thromboxane, and heart-produced prostacyclin in mrl mice. Am J Pathol 1984;117:125-130.

31 Mei C, Zheng F: Chronic inflammation potentiates kidney aging. Semin Nephrol 2009;29:555-568.

32 Bakan I, Laplante M: Connecting mtorc1 signaling to srebp-1 activation. Curr Opin Lipidol 2012;23:226234.

33 Lech M, Garlanda C, Mantovani A, Kirschning CJ, Schlondorff D, Anders HJ: Different roles of tir8/sigirr on toll-like receptor signaling in intrarenal antigen-presenting cells and tubular epithelial cells. Kidney Int 2007;72:182-192.

34 Leemans JC, Butter LM, Teske GJ, Stroo I, Pulskens WP, Florquin S: The toll interleukin-1 receptor (il-1r) 8/ single ig domain il-1r-related molecule modulates the renal response to bacterial infection. Infect Immun 2012;80:3812-3820.

35 Wullschleger S, Loewith R, Hall MN: Tor signaling in growth and metabolism. Cell 2006;124:471-484.

36 Fulco M, Cen Y, Zhao P, Hoffman EP, McBurney MW, Sauve AA, Sartorelli V: Glucose restriction inhibits skeletal myoblast differentiation by activating sirt1 through ampk-mediated regulation of nampt. Dev Cell 2008;14:661-673.

37 Fulco M, Sartorelli V: Comparing and contrasting the roles of ampk and sirt1 in metabolic tissues. Cell cycle 2008;7:3669-3679. 